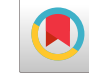

\title{
A Forty- Four Year - Old Immunized Woman with Maculopapular and Petechial Rash, Diagnosed as Measles: An Extremely Rare Presentation
}

\author{
Shahnaz Sali, ${ }^{1}$ Anita Yazdani, ${ }^{1}$ and Zahra Doosti ${ }^{1,}{ }^{*}$ \\ ${ }^{1}$ Infectious Diseases and Tropical Medicine Research Center, Shahid Beheshti University of Medical Sciences, Tehran, Iran \\ "Corresponding author: Zahra Doosti, Infectious Diseases and Tropical Medicine Research Center, Shahid Beheshti University of Medical Sciences, Tehran, Iran. E-mail: \\ z.doosti@sbmu.ac.ir \\ Received 2017 January 20; Revised 2017 February 03; Accepted 2017 February 03.
}

\begin{abstract}
Introduction: Measles is a highly contagious viral disease, which affects mostly children and rarely adults, which results in a more severe presentation. After a prodromal phase (malaise, anorexia, and the classic triad of conjunctivitis, cough, and coryza and a high fever), enanthem (Koplik spot) appears at buccal mucosa and then, typical erythematous maculopapular rash happens. There are several reports of atypical measles in the adults who have been immunized by killed type vaccine, therefore, prior history of vaccination with the original killed - virus among individuals who present with maculopapular rash and fever do not rule out measles. Case Presentation: Here we report a 44 - year - old woman with fever, chills, myalgia, odynophagia, exudative pharyngitis, bilateral parotitis, anterior cervical lymphadenopathy, tender splenomegaly, conjunctival suffusion and disseminated pruritic erythematous maculopapular, as well as a petechial rash all over her body. She was evaluated mainly for infectious mononucleosis, acute human immunodeficiency virus infection, and acute viral hepatitis, when all investigations were negative for possible diagnoses, although she mentioned immunization against measles, evaluations for measles were done and she had positive IgM antibody with high titer and also positive measles virus RT - PCR.

Conclusions: The aim of this report is to emphasize that physicians should be aware of the atypical measles syndrome and entertain the diagnosis of measles in adults with a febrile illness and rash in order to make the most accurate diagnosis and prevent complications.
\end{abstract}

Keywords: Measles, Fever, Exanthema, Adult, Vaccination

\section{Introduction}

Measles is a highly contagious viral disease with about 7 to 14 days of incubation period. It affects mostly the children, however, adults can rarely be afflicted. This disease is popular for its erythematous maculopapular rash, which often begins on the face and extends toward the trunk and eventually affects extremities including the soles and palms. The typical rash is appeared after prodromal phase. Koplik spots appear before the typical rash. People at a high risk for severe illness and complications from measles include Infants and children aged $<5$ years, adults aged $>20$ years, people with malnutrition and vitamin A deficiency, pregnant women and people with compromised immune system (such as leukemia and human immunodeficiency virus (HIV) infection) (1-3).

Despite the fact that measles typically occurs in children, when it affects adults, it results in a more severe illness, with the respiratory tracts infection, hepatitis, otitis media, and sinusitis as the most prevalent involvements
(4-6).

Measles is one of the preventable causes of death among young children, even though there is a safe and cost-effective vaccine for it. In 2015, there were 134200 deaths globally (about 15 deaths every hour). Measles vaccination resulted in a $79 \%$ drop in deaths caused by measles between 2000 and 2015 worldwide (3). From the year 2000 to 2015 , measles immunization resulted in approximately 20.3 million death preventions, therefore, efforts have issued efficient preventive measures such as global vaccination, in order to reduce the number of patients involved and prevent the consequent complications (7).

According to the epidemiology of measles, it is a rare infection in adults. However, with respect to several reports from atypical measles in the adults, physicians should be aware that this disease is not confined to children, and they should suspect measles in any one who presents with fever and rash, regardless of the age and history of previous vaccination. 


\section{Case Presentation}

Here we want to report a 44 - year - old woman who was referred to an emergency department in 2015 December, with complaint of fever, shaking chills, and myalgia, which began 7 days before. Sore throat, odynophagia, abdominal pain, mainly on the right and left upper quadrants, and redness of both eyes were added to her symptoms during that time. She did not have weight loss and constitutional symptoms. Dyslipidemia was the only underlying disease she has had, and no other comorbid conditions existed. She had already used atorvastatin $20 \mathrm{mg}$ once daily, however, except for 3 doses of ceftriaxone, which had been infused for her within 2 days before presentation, there was no newly started medication. She did not mention consumption of illicit drugs and alcohol. She was married and denied any extramarital relationships. Her husband was seronegative for human immunodeficiency virus (HIV) but she had multiple tattoos on her body surface.

On initial physical examination she was fully alert but critically ill, with intact orientation. Oral temperature was $39^{\circ} \mathrm{C}$, blood pressure was $90 / 60 \mathrm{mmHg}$, and she had 105 beats per minute pulse rate. Conjunctival suffusion was seen. Disseminated erythematous mild pruritic blanchable maculopapular rash and also petechial exanthem was visible all over her skin, including palms and soles, prominently on lower extremities, without any enanthem. Her face was plethoric and she had an exudative pharyngitis, bilateral parotitis, and anterior cervical lymphadenopathy. The chest examination, including rib cage, lungs, and heart was without pathologic findings. Tender splenomegaly was found on abdominal palpation. Liver was normal and no evidence of cirrhosis was evident. Peripheral vascular examination was normal.

The patient was admitted in the general infectious diseases ward and evaluations started with regard to her fever, rash, splenomegaly, pharyngitis, and lymphadenopathy.

The patient's rash and the results of initial laboratory investigations are available in Figure 1 and Table 1, respectively.

According to the results of physical and primary laboratory examinations, meningococcemia, Staphylococcal and Streptococcal toxic shock syndrome, infectious mononucleosis, drug eruption, acute retroviral syndrome (acute human immunodeficiency virus infection), acute viral hepatitis (including serum sickness), infective endocarditis, secondary syphilis, Hodgkin lymphoma (HL), and connective tissue diseases (including systemic lupus erythematous) were the main differential diagnoses.

Further laboratory investigations with respect to the mentioned impressions are available in Table 2.

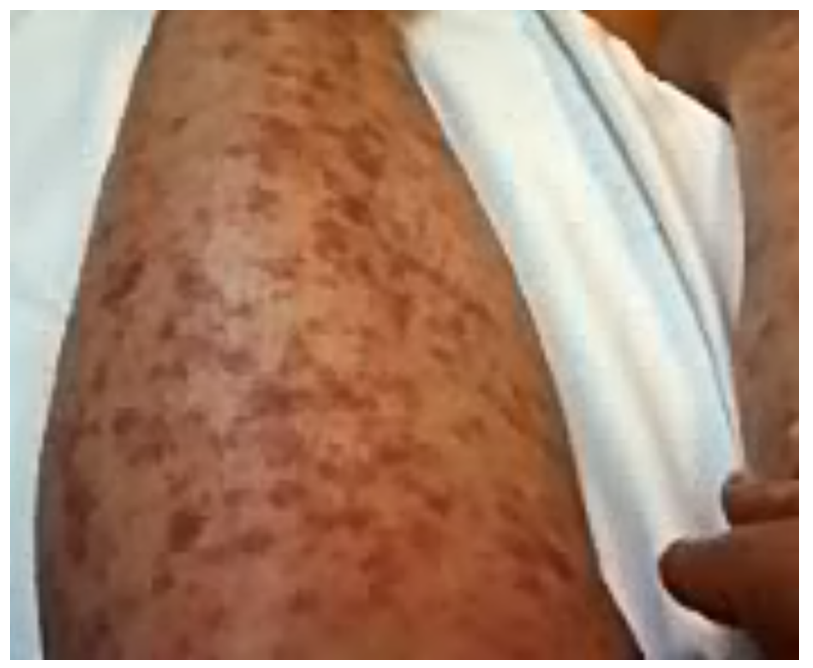

Figure 1. Patient's Blanchable Maculopapular and Petechial Rash

\begin{tabular}{lc}
\hline Table 1. Results of Initial Laboratory Investigations \\
\hline Parameter & \multicolumn{1}{c}{ Result } \\
\hline WBC count & $15 \times 10^{3} / \mu \mathrm{l}(\mathrm{lym}$ phocyte predominant) \\
\hline BUN & $20 \mathrm{mg} / \mathrm{dL}$ (normal) \\
\hline Serum Cr & $0.9 \mathrm{mg} / \mathrm{dL}$ (normal) \\
\hline Hb & $12.5 \mathrm{~g} / \mathrm{dL}$ \\
\hline MCV & $86.7 \mathrm{fL}$ \\
\hline Platelet & Platelet: $250 \times 10^{3} / \mu \mathrm{l}$ \\
\hline Sodium & $139 \mathrm{meq} / \mathrm{L}$ \\
\hline Potassium & $4.2 \mathrm{meq} / \mathrm{L}$ \\
\hline Venous blood gas & Normal \\
\hline Fasting blood sugar & $101 \mathrm{mg} / \mathrm{dL}$ \\
\hline C - reactive protein & 58 \\
\hline Erythrocytes sedimentation & 18 \\
rate & $1113 \mathrm{IU} / \mathrm{L}(\mathrm{elevated})$ \\
\hline PT & $1.2 \mathrm{mg} / \mathrm{dL}$ \\
\hline PTT & $0.3 \mathrm{mg} / \mathrm{dL}$ \\
\hline INR & $5 \mathrm{~g} / \mathrm{dL}$ \\
\hline AST & $13 \mathrm{~seconds}(\mathrm{normal})$ \\
\hline ALT & $35 \mathrm{~seconds}(\mathrm{normal})$ \\
\hline ALKPh & 1.1 \\
\hline Total serum bilirubin & $4000 \mathrm{IU} / \mathrm{L}(\mathrm{elevated})$ \\
\hline Direct bilirubin & \\
\hline Serum albumin & \\
\hline & \\
\hline
\end{tabular}

Chest X - ray revealed no pathologic finding. Trans - thoracic Echocardiography was negative for oscillating mass and pericardial effusion. Abdominal ultrasonography revealed normal - sized echogenic liver (grade II fatty liver), splenomegaly (spleen diameter: $13 \mathrm{~cm}$ ), and otherwise normal. 


\begin{tabular}{|c|c|}
\hline Parameter & Result \\
\hline Blood culture $\times 3$ ( 30 minutes apart) & Negative for any pathogens \\
\hline Fibrinogen and fibrinogen degradation product & Normal \\
\hline Rheumatologic investigations (including ANA and anti - dsDNA Ab) & Normal \\
\hline HIV - Ab (in two consecutive checks by ELISA method) & Non - reactive \\
\hline Venereal disease research laboratory (VDRL) & Negative \\
\hline Serum lactate dehydrogenase (LDH) & 1700 units (elevated) \\
\hline HbcAb & Non - reactive \\
\hline HBs Ag & Negative \\
\hline HBs Ab & $8 \mathrm{IU} / \mathrm{ml}$ \\
\hline HCV-Ab (EIA) & Non - reactive \\
\hline CMV Ab (IgM) & Negative \\
\hline CMV-PCR & Negative \\
\hline Mono test & Negative \\
\hline EBVVCA Ab (IgM) & Negative \\
\hline
\end{tabular}

All evaluations up to that time were inconclusive. According to her protracted high grade fever $\left(39^{\circ} \mathrm{C}\right.$ to $\left.41^{\circ} \mathrm{C}\right)$ in the 2 nd week of admission, before considering lymph node biopsy for determining whether Hodgkin lymphoma is the accurate diagnosis or not, and although she mentioned prior vaccination against measles, serology, and PCR for measles virus were done. Interestingly measles serology (IgM antibody) with ELISA method was positive with high titer and also measles virus RT - PCR was positive, therefore, lymph node biopsy was postponed and conservative therapies considered for her.

In the next week (i.e. the $3^{\text {rd }}$ week of admission), body temperature was gradually decreased, skin rashes began to evanesce and her general condition became better. She could eat solid foods without disturbance and discharged from the hospital with recommendation for follow - up.

On the next outpatient visit, a month later, she was well and also afebrile, without detectable lymphadenopathy, pharyngitis, and splenomegaly on examination.

\section{Discussion}

Although measles is a vaccine - preventable disease, it remains a leading cause of childhood mortality around the world and it causes outbreaks among populations with low vaccine coverage (1). Non - immunized individuals impose a significant risk of acquiring measles and its complications in themselves and their communities, as we know high levels of immunity is needed to prevent large outbreaks (7), therefore, emphasizing on the importance of vast and complete course of immunization seems necessary.

Besides this natural history and trying to make a global vaccination in order to reduce the burden of disease, there are various reports of adults who have been vaccinated with the killed virus in their childhood (i.e. before introduction of live attenuated vaccine in 1967), but developed atypical measles (8).

In addition to that, there are some other studies and reports from all over the world, which described individuals who were under coverage of live - attenuated vaccination; however, they also developed measles. As our patient was immunized with at least one dose of live - attenuated vaccine during her childhood, she did not mention any additional vaccine receipt.

In a study conducted by Caseries et al., 80 confirmed cases of measles (i.e. by compatible clinical presentations and also confirmatory laboratory tests), during the French 2010 - 2011 outbreak, were evaluated and among them, there were 10 people who had already been vaccinated, and 2 of them had received even 2 injections (6). Raman et al., described a 15 - year - old immunocompetent boy, presented with history of fever and exanthaematous maculopapular rash all over his face and body, except the lower extremities, who had positive IgM against measles virus (9). Chatterjee et al., reported a 28 - year - old Polish woman who had symptoms and signs mimicking human immunodeficiency virus seroconversion syndrome, and she was managed with this regard, however, she was finally diagnosed with measles. She had received 1 dose of measles vaccine but there was no evidence of the 2 nd dose receipt 
(10). In another study by Hahne et al., 8 health care workers were identified to have measles, 6 were vaccinated with the measles vaccine twice, 1 was vaccinated once, and 1 was unvaccinated. None of the twice - vaccinated cases had severe measles (11). An outbreak of measles reported by Rosen et al., in New York City, which was related to the individuals with evidence of immunity against measles. The index patient was vaccinated twice and 4 secondary patients had received either 2 doses of measles - containing vaccine or a previously positive IgG antibody against measles virus (12).

Similar reports from Iran were also notable. For example in a study by Izadi et al., a series of measles outbreaks during 2009 and 2010, in Chabahar, southeast of Iran, was reported (13). In addition, Moghadam et al., described a case series that consisted of 7 individuals who acquired measles, the 1st was an afghan refugee, however, the others were Iranian, and 4 individuals among them had documented vaccination history (even 1 dose or 2doses). Except 1 of them who was a 9 -year - old boy, the remaining 3 cases were under the age of 5 years. This means that vaccination protection may not be complete for all individuals who receive it (14).

The reports mentioned above raised the questions about the effectiveness of vaccination among general population. Which factors can be considered for these outbreaks and sporadic involvements? Whether reduction in antibodies and defect in other arms of immune system, or other causes?

With this aim, Ahmadi-Renani and his colleague evaluated 360 males ranging between 18 to 25 years old for their antibody levels against measles. They found that even in those who had been vaccinated, the IgG level was very low (15). This incomplete protection was also elucidated in a study conducted by Keshavarz et al., in which 53 medical students aged 20 to 30 years were included. A total of 36 of these 53 participants had received 2 doses of live attenuated measles vaccine according to the national program, during their childhood, and all of them had a history of Measles - Rubella vaccination during nationwide mass vaccination in 2003. There was a notable difference between the secondary immune responses (i.e. protective levels of IgG antibody against measles) induced by natural infection or immunization detected in this study and the immune responses detected in the post-campaign study, which was performed among individuals aged between 5 and 40 years in 2004 (79.2\% versus 97.4\%) (16). In addition, in a study by Wu et al., Cytotoxic T - Lymphocyte responses and also humoral responses against measles virus were measured. They demonstrated that nearly all individuals who developed measles generated acceptable humoral and cellular immune responses and only a few individuals had lack of sufficient cellular responses. They con- cluded that vaccine failure in those participated in their study was not due to improper host immune response to the virus (17).

According to the aforementioned studies, it can be concluded that supplementary immunizations with regular intervals, would augment cellular immune responses and maintain the sufficient levels of immunoglobulins, so that outbreaks can be prevented consequently.

Non - immunized adults are susceptible to acquisition of the measles virus, the same as immunized individuals with waning antibodies, in whom more severe illness and poor outcomes are expected (4-6). In our patient, in addition to high-grade fever and critical condition, a self - limited hepatitis also occurred.

As result, this study and similar reports underscore the need for meticulous clinical and laboratory investigations of suspected cases of measles, regardless of vaccination status, and we should be aware that this disease cannot be ruled out in previously immunized adults (either original killed vaccine or existing live - attenuated type), who are present with rash (exanthema) and fever.

\subsection{Conclusion}

In order to not impose a heavy economic and clinical burden on health care systems, and to prevent the consequent complications of measles, as a highly contagious illness, vaccination integrity with administering regular booster doses seems essential. In addition, applying the accurate and timely investigations for individuals who are suspected to have measles, even with history of prior immunization, would be justified.

\section{Acknowledgments}

The authors want to thank Dr. Davood Yadegarinia, the head of Infectious Diseases and Tropical Medicine Department, Shahid Beheshti University of Medical Sciences, for his kind general supervision. Also Ms. Zahra Arab Mazar for her assistance during submission process.

\section{Footnotes}

Authors' Contribution: Study concept and design: Dr. Shahnaz Sali, Dr. Anita Yazdani, Dr. Zahra Doosti; Acquisition of data: Dr. Anita Yazdani; Drafting of the manuscript: Dr. Anita Yazdani, Dr. Zahra Doosti; Editing the final manuscript: Dr. Shahnaz Sali, Dr. Zahra Doosti; Revision: Dr. Zahra Doosti.

Conflict of Interest: The authors have no conflict of interest.

Funding/Support: This case report had no financial support and sponsorship. 


\section{References}

1. Moss WJ, Griffin DE. Paramyxoviruses: Measles. Viral Infect Hum. 2014:537-52. doi: 10.1007/978-1-4899-7448-8_23.

2. Perry RT, Halsey NA. The clinical significance of measles: a review. J Infect Dis. 2004;189 Suppl 1:S4-16. doi: 10.1086/377712. [PubMed: 15106083].

3. World Health Organization . Measles. [cited November]. Available from: http://www.who.int/mediacentre/factsheets/fs286/en/.

4. Uzun O, Salk M, Findik S, Bayrak M, Erkan L. Severe measles pneumonia in an immunocompetent adult. Turk RespirJ. 2002;3:32-4.

5. Melenotte C, Cassir N, Tessonnier L, Brouqui P. Atypical measles syndrome in adults: still around. BMJ Case Rep. 2015;2015. doi: 10.1136/bcr2015-211054. [PubMed: 26400591].

6. Caseris M, Houhou N, Longuet P, Rioux C, Lepeule R, Choquet C, et al. French 2010-2011 measles outbreak in adults: report from a Parisian teaching hospital. Clin Microbiol Infect. 2014;20(4):O242-4. doi: 10.1111/1469-0691.12384. [PubMed: 24707854].

7. Centers for Disease Control . Prevention of Measles, Rubella, Congenital Rubella Syndrome, and Mumps, 2013: Summary Recommendations of the Advisory Committee on Immunization Practices (ACIP). MMWR Morb Mortal Wkly Rep. 2013;62(4):1-34.

8. Griffin DE, Pan CH. Measles: old vaccines, new vaccines. Curr Top Microbiol Immunol. 2009;330:191-212. [PubMed: 19203111].

9. Raman P, Hussaini SB, Saravanan P, Jeyaram K. Fever with Rash, an Alarm to the Physicians - A Case Report of Atypical Measles.JClin Diagn Res. 2016;10(2):OD12-3. doi: 10.7860/JCDR/2016/16170.7206. [PubMed: 27042506].
10. Chatterjee MT, Coleman M, Brook G, McCrea D. Measles mimicking HIV seroconversion syndrome: a case report.J Med Case Rep. 2010;4:41. doi: 10.1186/1752-1947-4-41. [PubMed: 20181109].

11. Hahne SJ, Nic Lochlainn LM, van Burgel ND, Kerkhof J, Sane J, Yap KB, et al. Measles Outbreak Among Previously Immunized Healthcare Workers, the Netherlands, 2014. J Infect Dis. 2016;214(12):1980-6. doi: 10.1093/infdis/jiw480. [PubMed: 27923955].

12. Rosen JB, Rota JS, Hickman CJ, Sowers SB, Mercader S, Rota PA, et al. Outbreak of measles among persons with prior evidence of immunity, New York City, 2011. Clin Infect Dis. 2014;58(9):1205-10. doi: 10.1093/cid/ciu105. [PubMed: 24585562].

13. Izadi S, Zahraie SM, Sartipi M. An investigation into a measles outbreak in southeast Iran. Jpn J Infect Dis. 2012;65(1):45-51. [PubMed: 22274157].

14. Moghadam M, Afsarkazerooni P, Ebrahimi M, Soltani M, Razmpoor A, Pirasteh E, et al. Measles outbreak in South of iran, where vaccine coverage was high: a case-series study. Iran J Public Health. 2014;43(3):37580. [PubMed: 25988100].

15. Ahmadi-Renani K, Ghorbani GA. Measles Sero-surveillance in Soldiers prior to Nation-wide Vaccination in Iran. Iran J Med Sci. 2015;30(2):803.

16. Keshavarz M, Nicknam MH, Tebyanian M, Shahkarami MK, Izad M. Anti-rubella, Mumps and Measles IgG Antibodies in Medical Students of Tehran University. Iran J Allergy Asthma Immunol. 2016;15(3):244-50. [PubMed: 27424140].

17. Wu VH, McFarland H, Mayo K, Hanger L, Griffin DE, Dhib-Jalbut S. Measles virus-specific cellular immunity in patients with vaccine failure. J Clin Microbiol.1993;31(1):118-22. [PubMed: 8417015]. 